\title{
Generation of orbital-angular-momentum-entangled biphotons in triangular quadratic waveguide arrays
}

\author{
Dmitry M. Markin, ${ }^{1}$ Alexander S. Solntsev,${ }^{2}$ and Andrey A. Sukhorukov ${ }^{2}$ \\ ${ }^{1}$ Quantum Electronics Division, Department of Physics, Lomonosov Moscow State University, Moscow 119992, Russia \\ ${ }^{2}$ Nonlinear Physics Centre, Research School of Physics and Engineering, Australian National University, Canberra ACT 0200, Australia
}

(Received 8 April 2013; published 10 June 2013)

\begin{abstract}
We suggest that closed-loop quadratic nonlinear waveguide arrays can be used as a compact interferometrically stable integrated source of discrete orbital-angular-momentum (OAM) entangled biphoton state. We describe analytically and numerically the process of biphoton generation through spontaneous parametric down-conversion in triangular waveguide arrays, and reveal that the generated biphoton quantum states can be precisely controlled by changing the pump phase and wavelength, including the case of photon-pair generation with pure OAM.
\end{abstract}

DOI: 10.1103/PhysRevA.87.063814

PACS number(s): 42.50.Tx, 42.65.Wi, 42.65.Lm

\section{INTRODUCTION}

Quantum teleportation [1-3] and quantum cryptography $[4,5]$ are among the most attractive applications of quantum entanglement [6], since they open the possibility of fully secure communication. The photon-pair entanglement in orbital-angular-momentum (OAM) basis was first experimentally demonstrated by Zeilinger $e t$ al. using the process of spontaneous parametric down-conversion (SPDC) [7]. Since then this topic has attracted growing attention due to the possibility of improved information transfer efficiency using this extra degree of freedom. It was also proposed that higher dimensional states using OAM may provide better security in comparison to standard quantum cryptography $[8,9]$.

The implementation of OAM entanglement with dynamically changeable holograms enabled the demonstrations of Bell inequality violation [10], spin-to-OAM transfer [11], and quantum cloning of OAM qubits [12]. Spatial light modulators also brought several advantages to quantum cryptography, such as large scale OAM encoding [13], entanglement between the angular position and OAM [14], and hybrid polarizationOAM-entangled states for greater quantum cryptography speeds [15]. More exotic ideas included OAM entanglement based on Bessel beams [16,17] and Fibonacci sequences [18].

Traditionally, the OAM-entangled quantum states are generated through SPDC in bulk quadratically nonlinear crystals [7]. With this approach, for a single pump beam, one generally obtains a set of photon pairs, for which the sum of momenta match the pump OAM according to the conservation law. For example, for a pump beam with zero OAM, the state emitted in the process of SPDC is represented by $|\Psi\rangle=P_{0,0}|0,0\rangle+P_{1,-1}|1,-1\rangle+P_{-1,1}|-1,1\rangle+$ $P_{2,-2}|2,-2\rangle+P_{-2,2}|-2,2\rangle+\cdots$, where $P_{0,0}, P_{1,-1}, P_{-1,1}$, etc., denote the corresponding probability amplitudes. To obtain a photon pair with a well defined OAM, it is necessary to prepare the pump beam with a certain OAM, and then filter out a particular photon state at the crystal output. However such optical schemes require a set of multiple optical elements and light modulators, for which interferometric stability has to be carefully controlled.

In this work we propose an integrated source of precisely controllable OAM-entangled biphoton states through SPDC in a closed-loop quadratic nonlinear waveguide array. Waveguide arrays can be fabricated in bulk crystals through femtosecond direct laser writing $[19,20]$, and such photonic circuits are intrinsically interferometrically stable. It was recently predicted that one-dimensional planar arrays of nonlinear waveguides can be used to generate through SPDC the photon pairs with nonclassical spatial correlations [21,22], which are more pronounced with respect to the correlations achieved during the propagation of photon pairs generated externally and coupled to a linear waveguide array [23]. On the other hand, biphoton evolution in linear closed-loop waveguide arrays can demonstrate new features compared to planar propagation [24], however the generation of photon pairs in nonlinear closed-loop waveguide arrays has not been studied so far. Here we apply the general theory of SPDC in multimode waveguides [25] and specifically waveguide arrays [21,26] to investigate a process of SPDC in a quadratic nonlinear triangular waveguide array and show the possibility of integrated discrete OAM-entangled biphoton state generation. We emphasize the importance of unusual spatial dispersion in these structures [27] and show that it allows for precise quantum state control, including pure OAM-entanglement generation without unwanted OAM terms.

The paper is organized as follows. In Sec. II, we formulate the theory and general solutions for photon-pair generation through SPDC in triangular quadratic nonlinear waveguide arrays. In Sec. III, we show how by choosing the pump profile and phase mismatch, it is possible to perform controlled generation of biphoton discrete vortex states with fixed angular moment or in entangled superposition states. Finally, we present conclusions in Sec. IV.

\section{PHOTON-PAIR GENERATION IN TRIANGULAR QUADRATIC NONLINEAR WAVEGUIDE ARRAYS}

We consider a triangular array of weakly coupled optical waveguides created in media with quadratic nonlinearity, as shown schematically in Fig. 1(a). We aim to describe the process of degenerate SPDC, where a pump beam photon spontaneously decays into a pair of identical signal and idler photons. Most commonly, at the short wavelengths of the pump beam the waveguide modes are strongly localized, such that the pump does not couple between the waveguides. However at the longer wavelengths of the down-converted photons the coupling can be essential [21]. Under such experimentally 
(a)

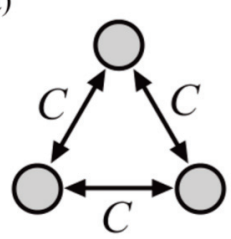

(b)

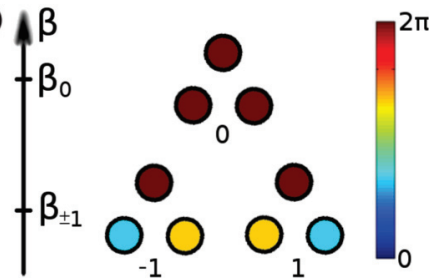

FIG. 1. (Color online) (a) Cross-section scheme of triangular waveguide array. (b) Supermode propagation constants and corresponding phase profiles.

feasible conditions, the photon-pair generation in quadratic waveguide arrays can be modeled with a discrete Schrödinger equation [26],

$$
\begin{aligned}
i \frac{d \Psi_{n_{s}, n_{i}}}{d z}= & -C\left[\Psi_{n_{s}+1, n_{i}}+\Psi_{n_{s}-1, n_{i}}\right. \\
& \left.+\Psi_{n_{s}, n_{i}+1}+\Psi_{n_{s}, n_{i}-i}\right] \\
& +i \sum_{n} d_{\mathrm{eff}} A_{n} \delta_{n_{s}, n} \delta_{n_{i}, n} \exp \left(i \Delta \beta^{(0)} z\right) .
\end{aligned}
$$

Here $\Psi_{n_{s}, n_{i}}$ is the biphoton wave function depending on two waveguide indices: for the signal photon $n_{s}$ and for the idler photon $n_{i} . A_{n}$ is the pump amplitude in the waveguide $n, d_{\text {eff }}$ is an effective nonlinear coefficient, and $\delta$ is a Kronecker delta function. $\Delta \beta^{(0)}$ is the phase mismatch between the pump field and generated biphoton state in a single waveguide, $\Delta \beta^{(0)}=$ $\beta_{p}-\left(\beta_{s}+\beta_{i}\right)$, where $\beta_{p, s, i}$ are the waveguide propagation constants of the pump, signal, and idler waves. $C$ is a coupling coefficient between the waveguide modes for the biphotons. In triangular waveguide arrays, we have $n_{s, i}=0,1,2$, and map other index numbers as $3 \rightarrow 0$ and $-1 \rightarrow 2$ according to the closed-loop boundary conditions.

To solve Eq. (1), it is convenient to first determine the linear eigenmodes of the triangular waveguide structure. For this purpose, we consider classical coupled-mode equations [28],

$$
i \frac{d E_{n}}{d z}+C\left(E_{n-1}+E_{n+1}\right)=0
$$

where $E_{n}$ is the complex field amplitude in the $n$th waveguide, and $E_{3}$ maps to $E_{0}$, and $E_{-1}$ maps to $E_{2}$ due to closed-loop boundary conditions. The eigenmode solutions of Eqs. (2) are $E_{n}^{(m)}(z)=\varepsilon_{n}^{(m)} \exp \left(i \beta_{m} z\right)$, where $m=0, \pm 1$ is the mode number, $\varepsilon_{n}^{(m)}=\exp (i 2 \pi m n / 3)$ are the mode profiles, and $\beta_{m}=2 C \cos (2 \pi m / 3)$ are the propagation constants: $\beta_{0}=$ $2 C, \beta_{ \pm 1}=-C$. The eigenmodes $E_{n}^{(m)}(z)$ represent discrete optical vortices [27], or states with OAM due to a helical structure of their phase profiles [see Fig. 1(b)]. Here the supermode number $m$ corresponds to the OAM.

We now seek a solution of Eq. (1) in the supermode representation,

$$
\Psi_{n_{s}, n_{i}}=\sum_{m_{s}=-1}^{1} \sum_{m_{i}=-1}^{1} \Phi_{m_{s}, m_{i}} e^{i 2 \pi m_{s} n_{s} / 3} e^{i 2 \pi m_{i} n_{i} / 3},
$$

where $\Phi_{m_{s}, m_{i}}$ are the amplitudes of states with the angular momenta $m_{s}$ and $m_{i}$ for the signal and idler photons, respectively. We perform a similar transformation of the pump

profile,

$$
A_{n}=\sum_{m=-1}^{1} F_{m} e^{i 2 \pi m n / 3}
$$

The resulting equation is

$\frac{d \Phi_{m_{s}, m_{i}}(z)}{d z}=i \beta_{m_{s}, m_{i}} \Phi_{m_{s}, m_{i}}(z)+e^{i \Delta \beta^{(0)} z} d_{\mathrm{eff}} F_{m_{s}+m_{i}} / 3$,

where

$$
\beta_{m_{s}, m_{i}}=2 C\left[\cos \left(\frac{2 \pi}{3} m_{s}\right)+\cos \left(\frac{2 \pi}{3} m_{i}\right)\right],
$$

and due to periodic boundary conditions we denote $F_{m} \equiv$ $F_{m+3}$.

The solution of Eq. (5) for photon pairs generated inside the structure, which corresponds to trivial initial condition $\Phi_{m_{s}, m_{i}}(0)=0$, is

$$
\begin{aligned}
\Phi_{m_{s}, m_{i}}(z)= & \frac{d_{\mathrm{eff}} z}{3} \exp \left[i\left(\beta_{m_{s}, m_{i}}+\Delta \beta^{(0)}\right) z / 2\right] \\
& \times F_{m_{s}+m_{i}} \operatorname{sinc}\left[\left(\beta_{m_{s}, m_{i}}-\Delta \beta^{(0)}\right) z / 2\right] .
\end{aligned}
$$

The real-space solution $\Psi_{n_{s}, n_{i}}(z)$ is then obtained by applying a transformation in Eq. (3).

We can now calculate the photon-pair correlation function,

$$
\Gamma_{n_{s}, n_{i}}=\left|\Psi_{n_{s}, n_{i}}\right|^{2} \text {. }
$$

Another useful characteristic of the biphoton state is the socalled nonclassicality calculated as [29]

$$
V_{n_{s}, n_{i}}=\frac{1}{3} \sqrt{\Gamma_{n_{s}, n_{s}} \Gamma_{n_{i}, n_{i}}}-\Gamma_{n_{s}, n_{i}} .
$$

Positive values of $V_{n_{s}, n_{i}}$ indicate true quantum behavior corresponding to the violation of Bell-like inequality.

In order to determine the degree of entanglement of the generated biphoton state, we perform the Schmidt decomposition. The state $\Psi_{n_{s}, n_{i}}$ has two discrete degrees of freedom $\left(n_{s}\right.$ and $n_{i}$ ), and its decomposition is defined as follows [30]:

$$
\Psi_{n_{s}, n_{i}}=\sum_{j=1}^{N} \sqrt{\lambda_{j}} \psi_{j n_{s}}^{(1)} \psi_{j n_{i}}^{(2)},
$$

where $N=3$ in our case of triangular waveguide array.

The orthonormal sets of vectors $\psi_{j}^{(1)}$ and $\psi_{j}^{(2)}$ are called Schmidt modes. The non-negative weight factors $\lambda_{j}$, called Schmidt coefficients, satisfy the normalization condition $\sum_{j=1}^{N} \lambda_{j}=1$. The Schmidt decomposition (10) is in fact a singular value decomposition [31]: $\Psi=U D V^{H}$, where $U$ and $V$ are unitary matrices formed from vectors $\psi_{j}^{(1)}$ and $\psi_{j}^{(2)}$ as columns (more strictly, $U_{n_{s} j}=\psi_{j n_{s}}^{(1)}, V_{n_{i} j}=\psi_{j n_{i}}^{(2) *}$ ), $V^{H}$ is the conjugate transpose of $V$, and $D$ is a diagonal matrix whose elements are the singular values of the matrix $\Psi$. These singular values give us Schmidt coefficients $\lambda_{j}: D_{j j}=\sqrt{\lambda_{j}}$. This decomposition was calculated numerically using standard routine from the LAPACK ++ library.

The number of nonzero Schmidt coefficients $\lambda_{j}$ in the decomposition (10) is called the Schmidt rank. In the particular case of only one nonzero Schmidt coefficient the state $\Psi_{n_{s}, n_{i}}$ is factorized as a product of two Schmidt modes, which means that the degrees of freedom $n_{s}$ and $n_{i}$ are not entangled. When 
TABLE I. Pump OAM (left column) and phase mismatch $\beta_{m_{s}, m_{i}}$ (central column) corresponding to the generated biphoton OAM (right column).

\begin{tabular}{ccc|}
\hline \hline $\begin{array}{c}\text { Pump state } \\
\left|m_{p}\right\rangle\end{array}$ & $\begin{array}{c}\text { Phase mismatch } \\
\beta_{m_{s} m_{i}}\end{array}$ & $\begin{array}{c}\text { Biphoton state } \\
\left|m_{s}, m_{i}\right\rangle\end{array}$ \\
\hline$|0\rangle$ & $4 \mathrm{C}$ & $|0,0\rangle$ \\
\hline$|0\rangle$ & $-2 \mathrm{C}$ & $|-1,1\rangle+|1,-1\rangle$ \\
\hline$|1\rangle \equiv|-2\rangle$ & $\mathrm{C}$ & $|0,1\rangle+|1,0\rangle$ \\
\hline$|1\rangle \equiv|-2\rangle$ & $-2 \mathrm{C}$ & $|-1,-1\rangle$ \\
\hline$|-1\rangle \equiv|2\rangle$ & $\mathrm{C}$ & $|0,-1\rangle+|-1,0\rangle$ \\
\hline$|-1\rangle \equiv|2\rangle$ & $-2 \mathrm{C}$ & $|1,1\rangle$ \\
\hline \hline
\end{tabular}

the Schmidt rank is more than 1, there are several nontrivial terms in Eq. (10) and the degrees of freedom are entangled.

\section{CONTROLLED GENERATION OF BIPHOTON DISCRETE VORTEX STATES}

\section{A. Phase matching and selection rules for biphoton vortices}

When a pump beam is launched into the triangular waveguide array with quadratic nonlinearity it can generate biphoton vortices, some of which are OAM-entangled states, through degenerate spontaneous parametric down-conversion. We summarize the possible biphoton states corresponding to the pump beams with different OAM in Table I. The left column $\left|m_{p}\right\rangle$ denotes a pump vortex state with orbital angular momentum $m_{p}$, the central column denotes phase mismatch $\Delta \beta^{0}$, and the right column $\left|m_{s}, m_{i}\right\rangle$ denotes resulting biphoton vortex states with orbital angular momenta $m_{s}$ and $m_{i}$.

As we noted earlier and show in Table I, the pump states $|1\rangle$ and $|-2\rangle$ are equivalent due to a finite number of waveguides in the array: Their phase profiles $\exp (i 2 \pi n / 3)$ and $\exp (-i 4 \pi n / 3)$ are identical. Thus the pump state $|1\rangle$ with angular momentum 1 can generate the biphoton state $|-1,-1\rangle$ with angular momentum -2 . Similarly, the pump state $|-1\rangle$ can generate the biphoton state $|1,1\rangle$. Further selection of generated biphoton states can be performed by choosing a certain phase mismatch, as we discuss below.

\section{B. Biphoton vortex states with certain angular momenta}

For a number of applications, efficient generation of biphoton vortex states with certain angular momenta $m_{s}$ and $m_{i}$ is essential. To archive this outcome, first it is important to ensure the phase matching between the pump state and the desired biphoton state:

$$
\Delta \beta=\Delta \beta^{(0)}-\beta_{m_{s}, m_{i}}=0 .
$$

The mismatch $\Delta \beta^{(0)}$ can be adjusted to satisfy this condition by tuning the pump wavelength.

Secondly it is necessary to appropriately choose the length of the waveguide array $L_{n}$ to suppress the generation of a biphoton state with undesired angular momenta $\tilde{m}_{s}$ and $\tilde{m}_{i}$ from the Table I:

$$
L_{n}=\frac{2 \pi n}{\mid \Delta \beta^{(0)}-\beta_{\widetilde{m}_{s}, \widetilde{m}_{i} \mid}}, \quad n=1,2,3, \ldots .
$$
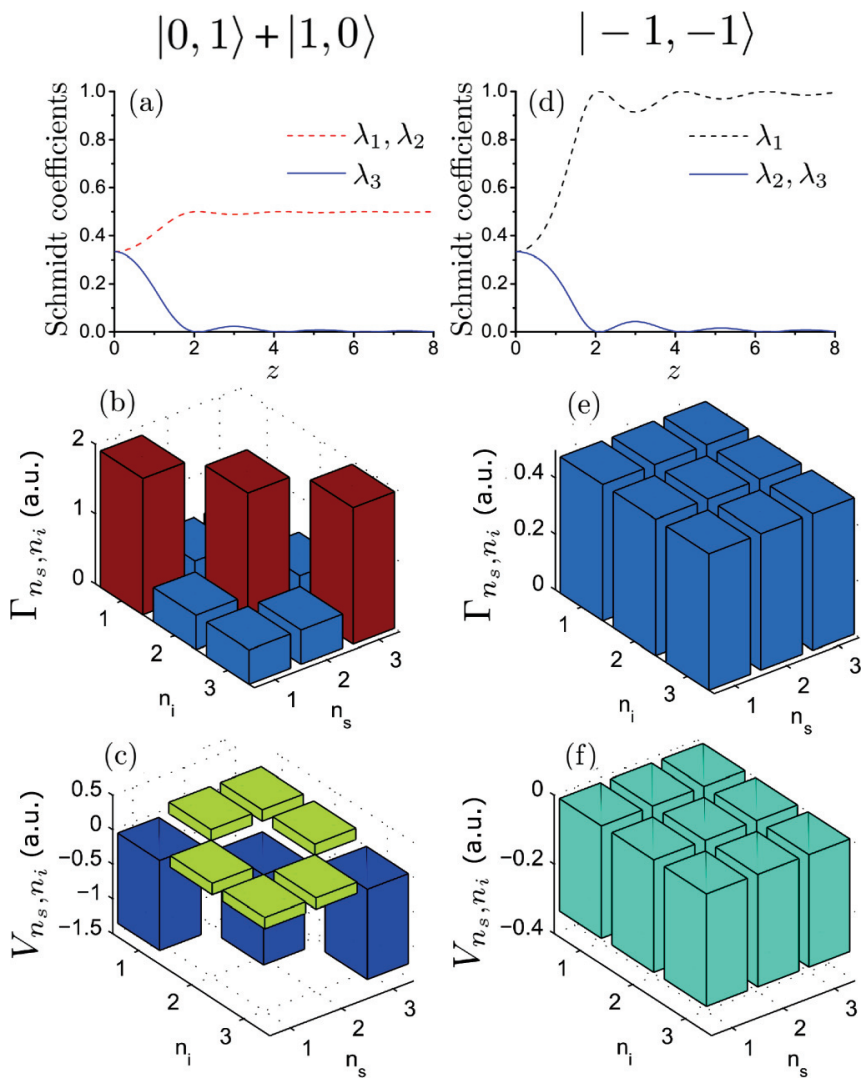

FIG. 2. (Color online) Generation of biphotons by a pump beam with angular momentum $m_{p}=1$ for different phase mismatches: (a)-(c) $\Delta \beta^{(0)}=C$ and (d)-(f) $\Delta \beta^{(0)}=-2 C$. (a), (d) Schmidt coefficients vs the propagation distance. (b), (e) Biphoton correlations and [(c), (f)] corresponding nonclassicality at $z=L_{1}=2 \pi / 3$. Normalized coefficients are $C=1, d_{\text {eff }}=1$.

To demonstrate the feasibility of this method we choose a pump vortex with angular momentum $m_{p}=1$, and define the other parameters as $C=1, d_{\text {eff }}=1$. We then consider two different phase mismatches: (i) at $\Delta \beta^{(0)}=C$ the phase-matching condition in Eq. (11) is satisfied for $|0,1\rangle+|1,0\rangle$ entangled biphoton state generation, and (ii) for $\Delta \beta^{(0)}=-2 C$ we can generate a $|-1,-1\rangle$ biphoton state. We plot the dependencies of the Schmidt coefficients on the propagation distance in Figs. 2(a) and 2(d). We also show the spatial biphoton correlations in Figs. 2(b) and 2(e) and the corresponding nonclassicality in Figs. 2(c) and 2(f) at the distance $z=2 \pi / 3$, corresponding to the first suppression length $\left(L_{1}\right)$ according to Eq. (12). At this distance, the undesired concurrent biphoton states are fully suppressed.

The state of two photons with different angular momenta $|0,1\rangle+|1,0\rangle$ has two nonzero Schmidt coefficients at $z=L_{1}$ [see Fig. 2(a)]. This means that the degrees of freedom of signal and idler photons are entangled. The quantum nature of this state is also clear from the nonclassicality matrix $V_{n_{s}, n_{i}}$ shown in Fig. 2(c) - it contains several positive elements.

The state with two identical angular momenta of both photons, $|-1,-1\rangle$, is simply a product state. It has only one nonzero Schmidt coefficient [Fig. 2(d)] at $z=L_{1}$, which means that the signal and idler photons are not entangled [see 

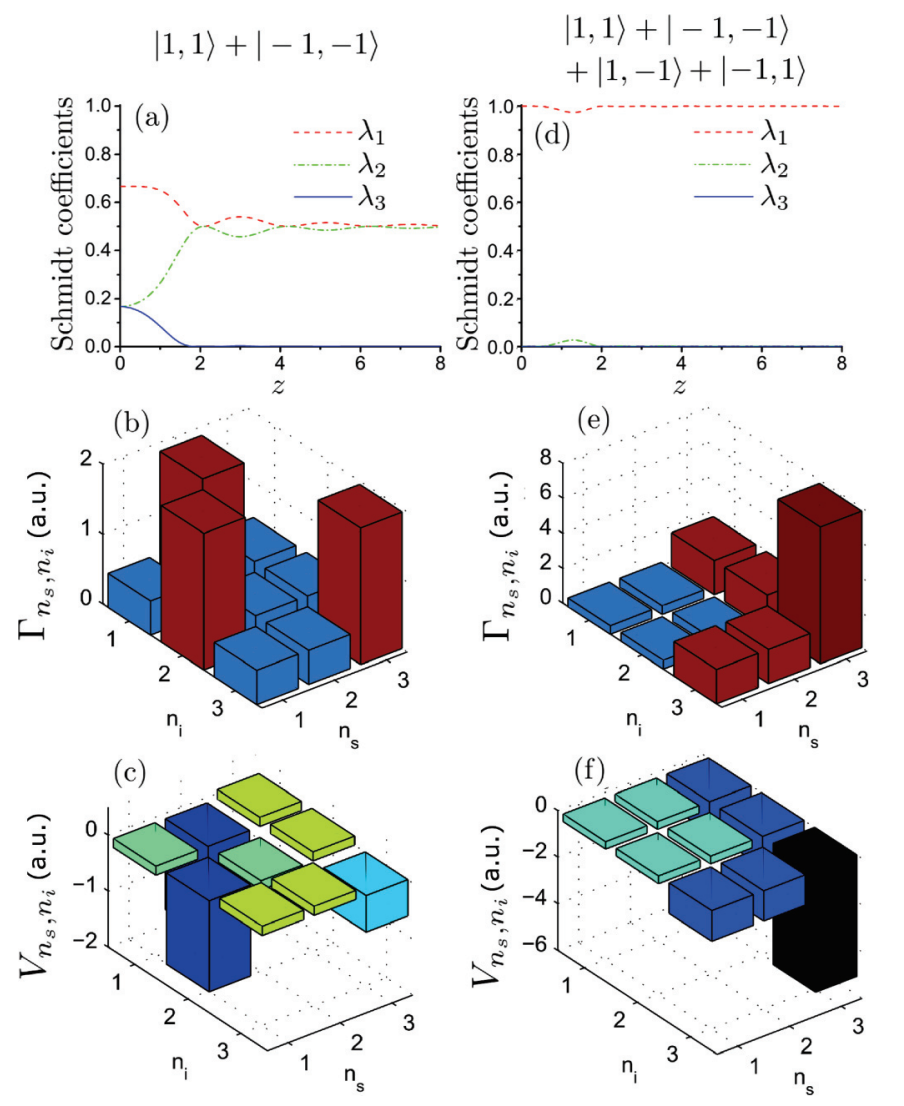

FIG. 3. (Color online) Generation of biphotons by a pump beam containing a superposition of several angular momenta: (a)-(c) $F_{1}=$ $F_{-1}=1, F_{0}=0$ and (d)-(f) $F_{1}=F_{-1}=F_{0}=1$. (a), (d) Schmidt coefficients. (b), (e) Biphoton correlations and [(c), (f)] corresponding nonclassicality at $z=L_{1}=2 \pi / 3$. Normalized coefficients are $C=$ $1, d_{\text {eff }}=1$. For all the plots the phase mismatch is $\Delta \beta^{(0)}=-2 C$, and the normalized coefficients are $C=1, d_{\mathrm{eff}}=1$.

correlations in Fig. 2(e)]. The nonclassicality matrix of this state, shown in Fig. 2(f), does not contain positive values.

\section{Superposition of biphoton vortex states}

We now demonstrate how to use the proposed scheme to generate the superposition of biphoton vortices in the phasematched regime. We see from Table I, that all states can have one of three different propagation constants. By tuning the phase matching with the pump beam, and selecting the pump beam profile, it is then possible to generate any superposition of biphoton states having the same propagation constant. At the same time, the other states can be suppressed when the distance satisfies Eq. (12).

We now illustrate the generation of the superposition of states $|1,1\rangle$ and $|-1,-1\rangle$. Using Table I, we determine the phase-matching condition as $\Delta \beta^{(0)}=\beta_{-1,-1}=\beta_{1,1}=$ $-2 C$, and the pump profile as a superposition of the right and left vortices: $F_{1}=F_{-1}=1$ and $F_{0}=0$, i.e., $A_{n}=$ $\exp (i 2 \pi n / 3)+\exp (-i 2 \pi n / 3)$ according to Eq. (4). We show in Figs. 3(a)-3(c) the evolution of the Schmidt coefficients, the biphoton correlations, and the corresponding nonclassicality at the distance $z=L_{1}$. We see that the generated state is entangled, with the nonclassicality matrix containing positive values and two nonvanishing Schmidt coefficients.

We also demonstrate in Figs. 3(d)-3(f) the generation of a biphoton state $|1,1\rangle+|-1,-1\rangle+|1,-1\rangle+|-1,1\rangle$, which is a superposition of all possible states for the propagation constant $\Delta \beta^{(0)}=-2 C$. Such state can be obtained using the pump in superposition of all angular momenta, $F_{1}=F_{-1}=$ $F_{0}=1$, i.e., $A_{n}=1+\exp (i 2 \pi n / 3)+\exp (-i 2 \pi n / 3)$. The Schmidt decomposition of this state contains only one mode at $z=L_{1}$ [Fig. 3(d)], i.e., it is not entangled. Indeed, although spatial correlations look nontrivial [Fig. 3(e)], the nonclassicality matrix does not contain positive elements [Fig. 3(f)].

\section{CONCLUSIONS}

To conclude, we have described analytically the generation of photon pairs through SPDC in triangular quadratic nonlinear waveguide arrays. We then formulated an approach for controlled biphoton discrete vortex state generation through tailoring the pump profile and phase-matching conditions. This method allows for flexible control of quantum statistics, including efficient generation of OAM-entangled states in the regime of phase matching. As the next step, it is an interesting open problem how to apply the concept of quasi-phasematching and design the spatial modulation of the sign of the nonlinear coefficient to efficiently generate a superposition of any states. We also anticipate that our theoretical analysis will stimulate experimental studies, as the triangular waveguide arrays can be fabricated using waveguide laser writing in nonlinear crystals.

\section{ACKNOWLEDGMENT}

This work was supported by the Australian Research Council, including Discovery Project DP130100135 and Future Fellowship FT100100160.
[1] D. Bouwmeester, J. W. Pan, K. Mattle, M. Eibl, H. Weinfurter, and A. Zeilinger, Nature (London) 390, 575 (1997).

[2] D. Boschi, S. Branca, F. De Martini, L. Hardy, and S. Popescu, Phys. Rev. Lett. 80, 1121 (1998).

[3] A. Furusawa, J. L. Sorensen, S. L. Braunstein, C. A. Fuchs, H. J. Kimble, and E. S. Polzik, Science 282, 706 (1998).

[4] A. K. Ekert, J. G. Rarity, P. R. Tapster, and G. Massimo Palma, Phys. Rev. Lett. 69, 1293 (1992).
[5] The Physics of Quantum Information: Quantum Cryptography, Quantum Teleportation, Quantum Computation, edited by D. Bouwmeester, A. K. Ekert, and A. Zeilinger (Springer, New York, 2000).

[6] A. Einstein, B. Podolsky, and N. Rosen, Phys. Rev. 47, 777 (1935).

[7] A. Mair, A. Vaziri, G. Weihs, and A. Zeilinger, Nature (London) 412, 313 (2001). 
[8] A. Vaziri, G. Weihs, and A. Zeilinger, Phys. Rev. Lett. 89, 240401 (2002).

[9] J. Leach, E. Bolduc, D. J. Gauthier, and R. W. Boyd, Phys. Rev. A 85, 060304 (2012).

[10] J. Leach, B. Jack, J. Romero, M. Ritsch-Marte, R. W. Boyd, A. K. Jha, S. M. Barnett, S. Franke-Arnold, and M. J. Padgett, Opt. Express 17, 8287 (2009).

[11] E. Nagali, L. Sansoni, F. Sciarrino, F. De Martini, L. Marrucci, B. Piccirillo, E. Karimi, and E. Santamato, Nat. Photonics 3, 720 (2009).

[12] E. Nagali, F. Sciarrino, F. De Martini, L. Marrucci, B. Piccirillo, E. Karimi, and E. Santamato, Phys. Rev. Lett. 103, 013601 (2009).

[13] M. McLaren, M. Agnew, J. Leach, F. S. Roux, M. J. Padgett, R. W. Boyd, and A. Forbes, Opt. Express 20, 23589 (2012).

[14] J. Leach, B. Jack, J. Romero, A. K. Jha, A. M. Yao, S. FrankeArnold, D. G. Ireland, R. W. Boyd, S. M. Barnett, and M. J. Padgett, Science 329, 662 (2010).

[15] E. Nagali and F. Sciarrino, Opt. Express 18, 18243 (2010).

[16] M. McLaren, M. Agnew, J. Leach, F. S. Roux, M. J. Padgett, R. W. Boyd, and A. Forbes, Opt. Express 20, 23589 (2012).

[17] S. Zhao, L. Gong, Y. Li, H. Yang, Y. Sheng, X. Dong, F. Cao, and B. Zheng, arXiv:1205.0851.

[18] D. S. Simon, N. Lawrence, J. Trevino, L. Dal Negro, and A. V. Sergienko, Phys. Rev. A 87, 032312 (2013).

[19] J. Burghoff, C. Grebing, S. Nolte, and A. Tunnermann, Appl. Phys. Lett. 89, 081108 (2006).
[20] M. Heinrich, A. Szameit, F. Dreisow, S. Doring, J. Thomas, S. Nolte, A. Tunnermann, and A. Ancona, Appl. Phys. Lett. 93, 101111 (2008).

[21] A. S. Solntsev, A. A. Sukhorukov, D. N. Neshev, and Y. S. Kivshar, Phys. Rev. Lett. 108, 023601 (2012).

[22] A. S. Solntsev, A. A. Sukhorukov, D. N. Neshev, and Y. S. Kivshar, Opt. Express 20, 27441 (2012).

[23] A. Peruzzo, M. Lobino, J. C. F. Matthews, N. Matsuda, A. Politi, K. Poulios, X. Q. Zhou, Y. Lahini, N. Ismail, K. Worhoff, Y. Bromberg, Y. Silberberg, M. G. Thompson, and J. L. O'Brien, Science 329, 1500 (2010).

[24] J. O. Owens, M. A. Broome, D. N. Biggerstaff, M. E. Goggin, A. Fedrizzi, T. Linjordet, M. Ams, G. D. Marshall, J. Twamley, M. J. Withford, and A. G. White, New J. Phys. 13, 075003 (2011).

[25] A. Christ, K. Laiho, A. Eckstein, T. Lauckner, P. J. Mosley, and C. Silberhorn, Phys. Rev. A 80, 033829 (2009).

[26] M. Grafe, A. S. Solntsev, R. Keil, A. A. Sukhorukov, M. Heinrich, A. Tunnermann, S. Nolte, A. Szameit, and Y. S. Kivshar, Sci. Rep. 2, 562 (2012).

[27] A. S. Desyatnikov, M. R. Dennis, and A. Ferrando, Phys. Rev. A 83, 063822 (2011).

[28] F. Lederer, G. I. Stegeman, D. N. Christodoulides, G. Assanto, M. Segev, and Y. Silberberg, Phys. Rep. 463, 1 (2008).

[29] Y. Bromberg, Y. Lahini, R. Morandotti, and Y. Silberberg, Phys. Rev. Lett. 102, 253904 (2009).

[30] A. Ekert and P. L. Knight, Am. J. Phys. 63, 415 (1995).

[31] G. H. Golub and C. F. Van Loan, Matrix Computations, 3rd ed. (The Johns Hopkins University Press, Baltimore, 1996). 